\title{
Microdissection of guinea pig extraocular muscles
}

\author{
JI-BO ZHOU ${ }^{1,2}$, SHENGFANG GE ${ }^{2}$, PING GU ${ }^{2}$, DUO PENG ${ }^{1}$, GUO-FU CHEN ${ }^{1}$, MIAO-ZHEN PAN ${ }^{1}$ and JIA QU ${ }^{1}$ \\ ${ }^{1}$ Wenzhou Medical College, School of Ophthalmology and Optometry, Zhejiang; ${ }^{2}$ Department of Ophthalmology, \\ Ninth People's Hospital, Shanghai Jiaotong University School of Medicine, Shanghai, P.R. China
}

Received July 11, 2011; Accepted August 3, 2011

DOI: $10.3892 /$ etm.2011.341

\begin{abstract}
The guinea pig, a widely used experimental animal, has been used in myopia research in recent years. The structure of the extraocular muscles is important in research on eyeball movement, regulation of movement, binocular vision and surgical intervention. In this study, the anatomy and the structure of the extraocular muscles of guinea pigs were investigated. Five guinea pig eyes were dissected under a surgical microscope immediately after sacrifice, and an additional five were fixed in $10 \%$ formaldehyde solution and dissected under a surgical microscope 1 week after sacrifice. The guinea pig eye has seven extraocular muscles: two medial rectus muscles, one superior rectus muscle, one inferior rectus muscle, one superior oblique muscle and one inferior oblique muscle. The retractor bulbi muscle fibers surround the optic nerve longitudinally and insert circumferentially into the posterior pole of the eyeball. The lateral rectus was not found. Our results showed that there is a disparity between the structure of guinea pig extraocular muscles and that of humans.
\end{abstract}

\section{Introduction}

The guinea pig, a widely used experimental animal, plays an important role in medical and biological experiments. The structure of the guinea pig eyeball is similar to humans and therefore guinea pig eyes have been used in myopia research in recent years. For example, Howlett and McFadden showed that changes in a guinea pig eyeball and refraction after defocusing are similar to other mammals (1). Lu et al (2) and Zhou et al (3) induced myopia by applying a facemask to intervene in the developing guinea pig eyes. The formation of myopia in humans is significantly related to the development of extraocular muscles (4). Von Graefe cited the activity of the extraocular muscles, specifically the horizontal recti and the medials, as myopigenic owing to their compression of the eye in convergence (5). More recently, Greene reviewed the potential effects of accommodation, vitreous pressure, conver-

Correspondence to: Dr Jia Qu, Wenzhou Medical College, School of Ophthalmology and Optometry, Wenzhou, Zhejiang, P.R. China E-mail: jqu@wz.zj.cn; zhoujibo1000@yahoo.com.cn

Key words: extraocular muscles, guinea pig, microdissection gence and the extraocular muscles upon the human eye, and concluded that convergence and the tension in the extraocular muscles are of greatest importance because of their affect upon vitreous pressure. They specifically implicated the oplique muscles in this regard $(6,7)$. The structure and function of the extraocular muscles is important in research on eyeball movement, regulation of movement, binocular vision and surgical intervention. Investigation of extraocular muscle anatomy and structure is important in the research of myopia development. To our knowledge, there are only a few publications on the microscopic anatomy of the extraocular muscles of guinea pigs (8). Determining the anatomy of the extraocular muscles of the guinea pig will set the foundation for ophthalmological and optometric research that uses guinea pig eyes as models.

\section{Materials and methods}

The present study used 10 -week-old British guinea pigs $(\mathrm{n}=10)$ weighing between 400 and $450 \mathrm{~g}$. Those with significant maldevelopment were eliminated. The guinea pigs were obtained from the Shanghai Experimental Animal Center of the Chinese Academy of Sciences. Rearing and treatment protocol was approved by the Animal Ethics Committee of the Wenzhou Medical College.

The guinea pigs were sacrificed by an overdose of anesthesia with an intraperitoneal injection of $1.5 \mathrm{ml}$ of a mixture of $1.2 \mathrm{ml} 10 \%$ ketamine hydrochloride, $0.8 \mathrm{ml} 2 \%$ xylazine hydrochloride and $8.0 \mathrm{ml}$ sterile saline. After decapitation, the guinea pig heads were placed under a microscope. The eye fissure was expanded by $1 \mathrm{~cm}$ on the nasal and temporal side. The skin around the eye was cut along the conjunctiva leaving the eye fully exposed. The membrane of the eyeball was removed close to the corneal limbus, and the membrane bag was separated exposing the connective tissue on the eyeball surface by pulling on the eyeball. The extraocular muscles were isolated by marking the original points and the points of insertion, and then removing the orbital septum and fat. The heads of the remaining five pigs were removed and their skin was removed after being sacrificed. The skull was split along the midline and placed into $10 \%$ neutral formaldehyde solution for 1 week. The optic nerve from the optic chiasm was identified under the microscope and the bone tissue was peeled off with a hemostat. The bony orbital wall was removed, the periosteum opened and the muscle tissue was identified. The fat tissue and orbital septum were removed from the eye socket, and the number of muscles and the points of origin 
and insertion of the extraocular muscles were determined. The retractor bulbi muscle was confirmed by paraffin sections and tissue $\mathrm{H} \&$ E staining.

\section{Results}

The guinea pig has seven extraocular muscles: two medial rectus muscles, one superior rectus muscle, one inferior rectus muscle, one superior oblique muscle, one inferior oblique muscle and one retractor bulbi muscle (Figs. 1-6). Two medial rectus muscles originate from the optic foramen around the optic nerve, located on the back temporal side of the orbit (Fig. 4). They are parallel with separate origins and insertions. Two medial rectus muscles are located on the nasal side of the eyeball after passing around the equator of the eyeball from the nasal side along the wall. The insertion lines are situated $1.5 \mathrm{~mm}$ from the nasal limbus and parallel to the limbus. The superior rectus muscle travels from the back temporal side of the optic foramen, while the inferior rectus muscle travels from the back temporal side of the optic foramen and inserts $1.5 \mathrm{~mm}$ inferior to the limbus. The superior and inferior oblique muscles both originate from the medial orbital wall following a straight course and are behind the insertions of the superior rectus muscles and inferior rectus muscles. The insertion line of the superior rectus muscles and superior oblique muscle creates a $45^{\circ}$ angle. The insertion of the inferior rectus and inferior oblique muscles creates a $20^{\circ}$ angle. Among the six muscles that insert on the aspect near the limbus, the length of the two medial rectus muscles and tendons were the longest of all the extraocular muscles, while the superior rectus muscle was relatively short. The retractor bulbi muscle originates from the optic foramen within the origins of the rectus muscles. It surrounds the optic nerve longitudinally and is inserted circumferentially into the posterior pole of the eyeball around the optic nerve head (Figs. 5 and 6).

\section{Discussion}

The data revealed that the three color breed of British guinea pigs has two medial rectus muscles, but no lateral rectus muscle. The superior oblique muscle track does not have a trochlea structure, which is present in the human eye. The specimens in our study confirmed the tracks of the extraocular muscles and the relative location to the eyeball. It is generally agreed that most mammals, such as tigers and lions, have four rectus and two oblique muscles (10). The origins of the four rectus muscles are close to the optic nerve behind the orbit. The location of the eye shank and two oblique muscles originate from the front of the orbit. The results of this study indicate that the number and origins of guinea pig extraocular muscles have the characteristics of most vertebrates. The reports by Cooper and Schiller suggest that the guinea pig has four rectus muscles (medial, lateral, superior and inferior), two oblique muscles and one retractor bulbi muscle (8). The four rectus muscles originate from the optic nerve around the orbit's apex; the two oblique muscles originate from the medial wall of the orbit, but without the pulley structure observed in the human eye on the superior oblique muscle (8). Their descriptions are different from the results presented here. There are five types of guinea pigs and accounting for the five most common

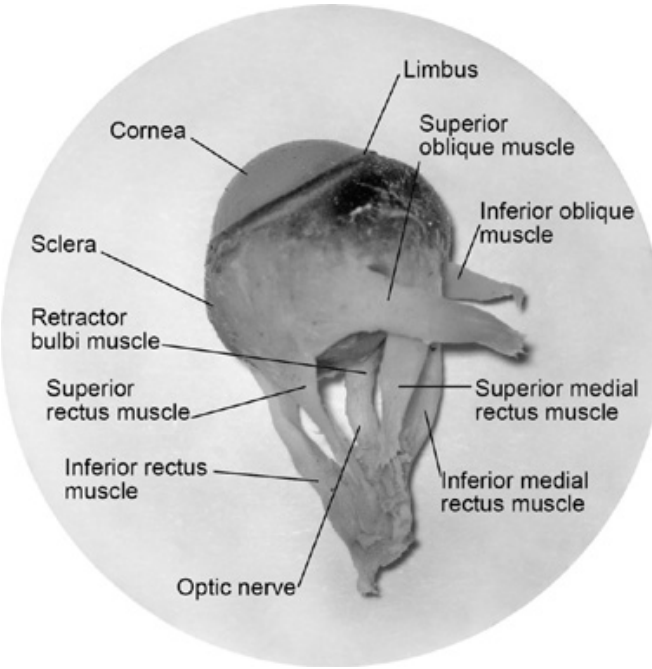

Figure 1. Cranial view of the left eyeball.

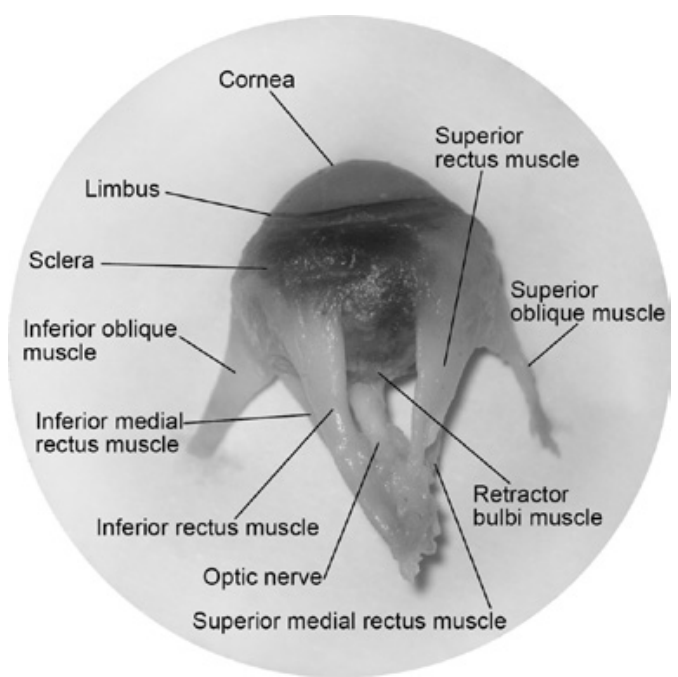

Figure 2. Temporal view of the left eyeball. The lateral rectus muscle was not found between the superior rectus muscle and the inferior rectus muscle.

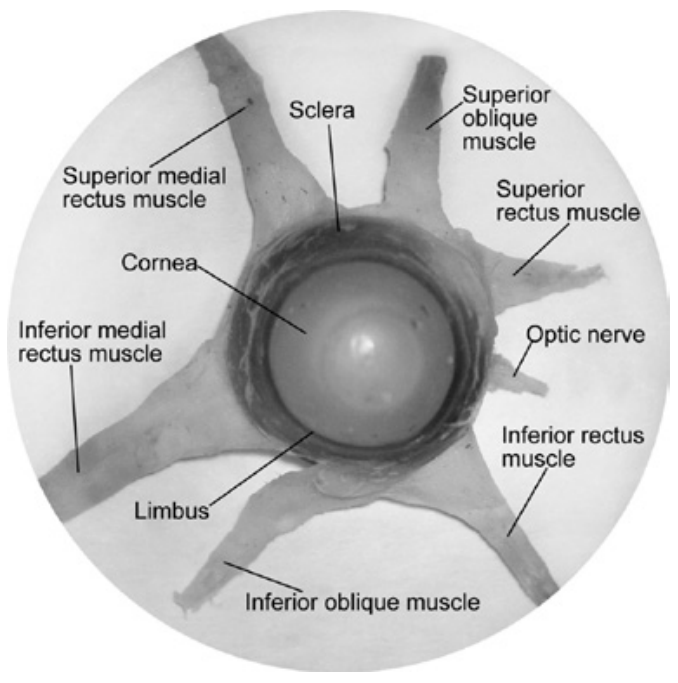

Figure 3. Front view of the left eyeball. There are two medial rectus muscles (the superior media muscle and the inferior media muscle), two oblique muscles (the superior oblique muscle and the inferior oblique muscle), the superior rectus muscle and the inferior muscle shown in this image. 


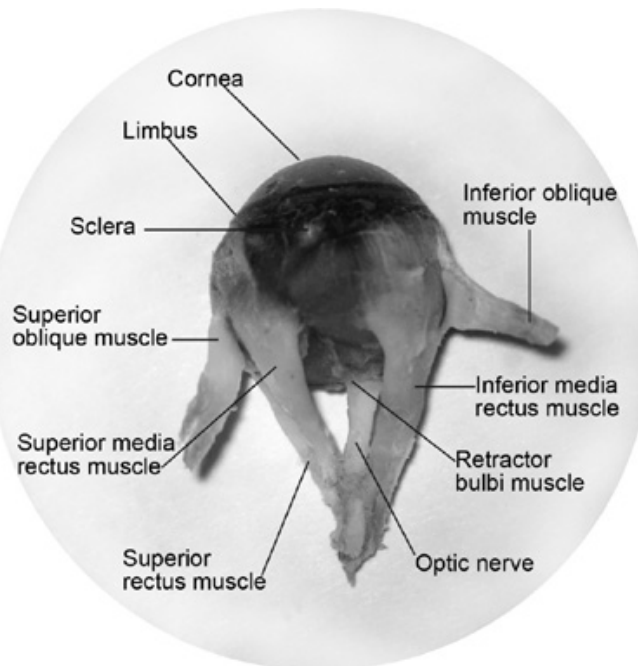

Figure 4. Nasal view of the left eyeball. There are two medial rectus muscles between the inferior oblique muscle and the superior oblique muscle.

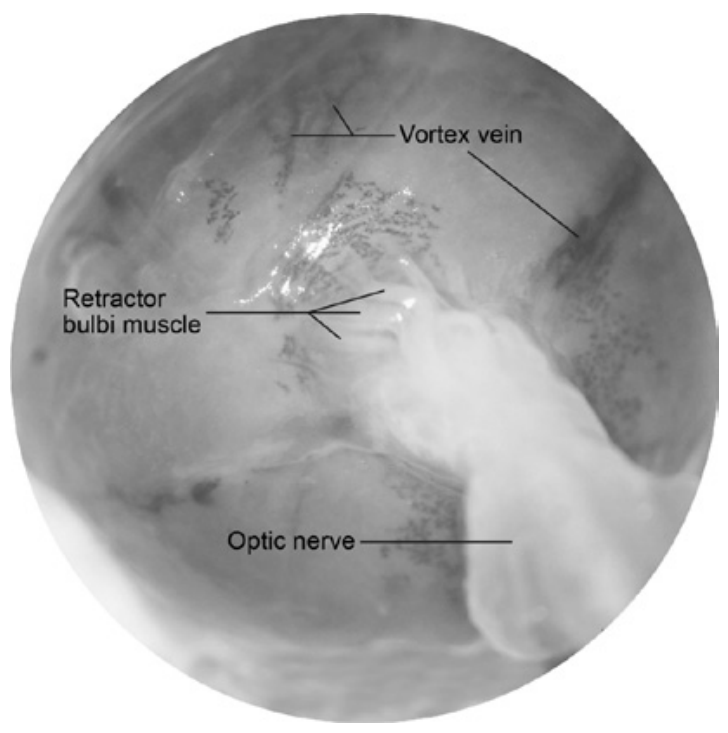

Figure 5. Retractor bulbi muscle around the optic nerve.

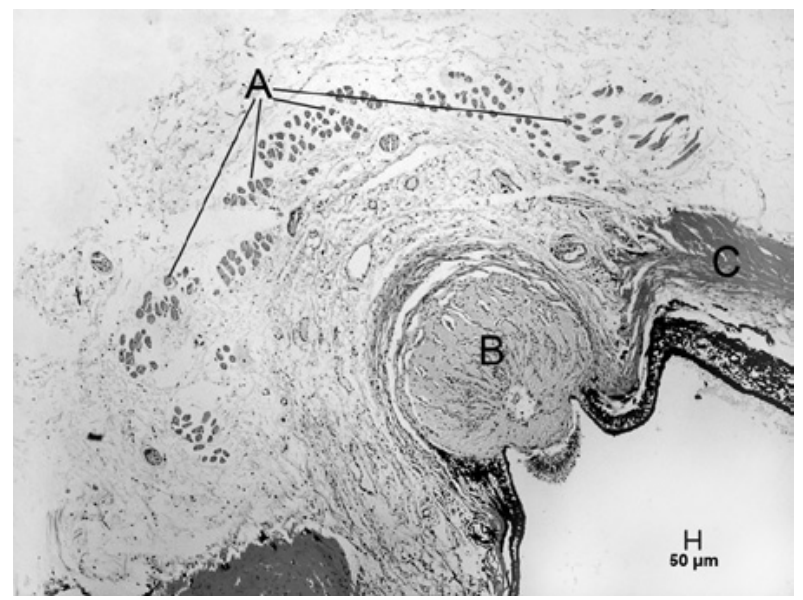

strains of guinea pigs [short-haired (British and American species), Dunkan-Hartley, Hartly, 2 strain and 13 strain], we do not consider that the disparities between previous reports and the results of this study are explained by species differences.

Typically, the eyes of certain lower level animals are located on the sides of the head, thus giving a large visual field, but no three-dimensional vision. During evolution, the eyes of higher level animals have moved to the front of the head allowing for three-dimensional vision. The optical axis angle of the guinea pig ranges from 103 to $110^{\circ}$; binocular vision ranges from 20 to $63^{\circ}$ and the combined visual field is between 325 and $340^{\circ}$. By contrast, the optical axis angle of the human eye is $0^{\circ}$; binocular vision is between 140 to $160^{\circ}$ and the combined visual field is between 180 and $190^{\circ}$. The guinea pig's combined visual field is close to $360^{\circ}(9,10)$, and therefore the lateral rectus muscle is not required. The optic nerve of the guinea pig is located near the temporal side from the anatomical structure of the superior and inferior rectus muscles, and the origin is on the back of the eyeball's temporal side. Therefore, the superior and inferior rectus muscles provide sufficient movement of the eyeball. Guinea pig eyes are located on two sides of the head; therefore, binocular vision is relatively narrow. The two medial rectus muscles are conducive to broadening binocular vision and achieving three-dimensional vision.

In conclusion, the anatomy of the guinea pig extraocular muscles is different from that of humans. There are two medial rectus muscles without a lateral rectus muscle, which is consistent with eyeball location and guinea pig living habits.

\section{Acknowledgements}

This study was supported by the Shanghai Leading Academic Discipline Project (S30205) and the Science and Technology Foundation of Shanghai Jiaotong University School of Medicine.

\section{References}

1. Howlett MH and McFadden SA: Form-deprivation myopia in the guinea pig (Cavia porcellus). Vision Res 46: 267-283, 2006.

2. Lu F, Zhou X, Zhao H, Wang R, Jia D, Jiang L, Xie R and Qu J: Axial myopia induced by a monocularly-deprived facemask in guinea pigs: a non-invasive and effective model. Exp Eye Res 82: 628-636, 2005.

3. Zhou X, Lu F, Xie R, Jiang L, Wen J, Li Y, Shi J, He T and Qu J: Recovery from axial myopia induced by a monocularly deprived facemask in adolescent (7-week-old) guinea pigs. Vision Res 47: 1103-1111, 2007.

4. Curtin BJ: The Myopias. Harper \& Row Publishers, Philadelphia, p104, 1985.

5. Von Graefe A: Beitrage zur Physiologie und Pathologie der schiefen Augenmuskeln. Albrecht Von Graefes Arch Ophthalmol 3: 277, 1857.

6. Greene PR: Myopia and the extraocular muscles. Doc Ophthalmol 8: 163, 1981.

7. Greene PR: Mechanical aspects of myopia. Master's thesis. Harvard University, 1978.

8. Cooper G and Schiller AL: Anatomy of the Guinea Pig. Harvard University Press, Cambridge, MA, pp369-389, 1975.

9. Duke-Elder S (ed): The Eye in Evolution. System of Ophthalmology. Vol. 1. Henry Kimpton Press, London, p672, 1976.

10. Prince Jack H: Comparative Anatomy of the Eye. Charles C Thomas Publisher, Illinois, pp27-29; 286-298, 1956.

Figure 6. Tissue section of the optic nerve and tractor bulbi muscle. (A) Muscle fibers; (B) optic nerve; (C) sclera. 